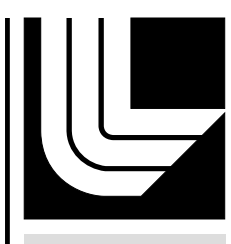

LAWRENCE LIVERM ORE N A TIO NAL LABORATORY

Convective Heating of the LIFE Engine Target During Injection

D. S. Holdener, M. S. Tillack, X. R. Wang

October 25, 2011 
This document was prepared as an account of work sponsored by an agency of the United States government. Neither the United States government nor Lawrence Livermore National Security, LLC, nor any of their employees makes any warranty, expressed or implied, or assumes any legal liability or responsibility for the accuracy, completeness, or usefulness of any information, apparatus, product, or process disclosed, or represents that its use would not infringe privately owned rights. Reference herein to any specific commercial product, process, or service by trade name, trademark, manufacturer, or otherwise does not necessarily constitute or imply its endorsement, recommendation, or favoring by the United States government or Lawrence Livermore National Security, LLC. The views and opinions of authors expressed herein do not necessarily state or reflect those of the United States government or Lawrence Livermore National Security, LLC, and shall not be used for advertising or product endorsement purposes.

This work performed under the auspices of the U.S. Department of Energy by Lawrence Livermore National Laboratory under Contract DE-AC52-07NA27344. 


\title{
CONVECTIVE HEATING OF THE LIFE ENGINE TARGET DURING INJECTION
}

\author{
D. S. Holdener, M. S. Tillack and X. R. Wang \\ Mechanical and Aerospace Engineering Department, Center for Energy Research, \\ University of California, San Diego, La Jolla, CA 92093-0438, dainholdener@gmail.com
}

\begin{abstract}
Target survival in the hostile, high temperature xenon environment of the proposed Laser Inertial Fusion Energy (LIFE) engine is critical. This work focuses on the flow properties and convective heat load imposed upon the surface of the indirect drive target while traveling through the xenon gas. While this rarefied flow is traditionally characterized as being within the continuum regime, it is approaching transition where conventional CFD codes reach their bounds of operation. Thus ANSYS, specifically the Navier-Stokes module CFX, will be used in parallel with direct simulation Monte Carlo code DS2V and analytically and empirically derived expressions for heat transfer to the hohlraum for validation. Comparison of the viscous and thermal boundary layers of ANSYS and DS2V were shown to be nearly identical, with the surface heat flux varying less than $8 \%$ on average. From the results herein, external baffles have been shown to reduce this heat transfer to the sensitive laser entrance hole (LEH) windows and optimize target survival independent of other reactor parameters.
\end{abstract}

\section{INTRODUCTION}

The LIFE engine utilizes multiple laser beams to compress fuel capsules suspended within indirect drive hohlraum targets injected into a fusion chamber. The spherical compression of the fuel capsules filled with deuterium and tritium will result in ignition, releasing ions, $\mathrm{x}$-rays, and high-energy neutrons. While the neutrons are absorbed by a fusion blanket surrounding the chamber to produce electricity, xenon gas will fill the chamber to absorb damaging ion and x-ray radiation.

In order to produce enough power to be competitive with modern fission energy plants ( $1 \mathrm{GW})$, the LIFE engine is designed to engage 10-20 targets per second. These continual thermonuclear burns are expected to keep the xenon gas upwards of $8000 \mathrm{~K}$, however, the fusion targets must now survive a flight of 6 meters through a very hostile environment.

Previous fusion energy design studies have used xenon or similar gases as a buffer to reduce ion and x-ray radiation within the target chamber. The majority of these studies were performed utilizing direct drive targets where the gas pressure of the chamber gas was limited to the 1-100 mtorr range to prevent excessive convective heating of the cryogenic deuterium-tritium fuel layer (Ref. 1-2). Placing the fuel capsule within the hohlraum acts as a buffer to delay this heating, and thus has enabled the LIFE design to increase the xenon density by approximately an order of magnitude.

Consequently has changed the nature of the flow field. The flight of the targets moved from the transition to the continuum regime, thus making traditional NavierStokes CFD methods applicable to this analysis.

The density of the chamber gas remains sufficiently low such that a direct simulation Monte Carlo solution is tractable on a modern PC platform, and will be used in parallel to investigate the flow field and heat transfer to the target. The resulting surface heat fluxes will then be compared with empirically and analytically derived correlations of heat transfer for their validation.

\section{TARGET FLIGHT CONDITIONS}

Since the LIFE engine is still in its early stages of design, target and chamber specification have not been finalized. TABLE I illustrates the latest design conditions relevant for convective heating of the targets transversing the target chamber. A simplified illustration of the current target design, known as the LIFE.2 hohlraum, is shown in Fig. 1. Note, P2 radiation shields, the tent holding the fuel capsule as well as the fuel capsule itself has been omitted within this illustration.

\section{II.A. Xenon Transport Properties}

The viscosity and thermal conductivity of xenon has been estimated using kinetic theory, which can be used until the onset of ionization. Using the Lennard-Jones potential for modeling intermolecular forces between particles, the Chapman-Enskog theory yields expressions for viscosity $(\mu)$ and thermal conductivity (k) as a function of temperature and are given by Eq. (1) and (2) (Ref. 3).

$$
\mu=2.6693 e^{-5} \frac{\sqrt{M T}}{\sigma^{2} \Omega_{\mu}}
$$


TABLE I. Target Flight Conditions (TFC)

\begin{tabular}{cc}
\hline Hohlraum & \\
\hline initial temperature & $17 \mathrm{~K}$ \\
injection velocity & $250 \mathrm{~m} / \mathrm{s}$ \\
spin rate & $15,000 \mathrm{RPM}$ \\
wall material & lead \\
radius, OD & $9.9 \mathrm{~mm}$ \\
length & $15.4 \mathrm{~mm}$ \\
wall thickness & (variable, see Fig. 1) \\
LEH window material & graphene \\
LEH window thickness & $0.5 \mu \mathrm{m}$ \\
Chamber & \\
radius & $6 \mathrm{~m}$ \\
gas species & $\times e n o n$ \\
gas temperature & $8000 \mathrm{~K}$ \\
gas density & $2.75 \times 10^{22} / \mathrm{m}^{3}$ \\
gas pressure at Tg & $3037 \mathrm{~Pa}(23$ torr $)$ \\
\hline
\end{tabular}

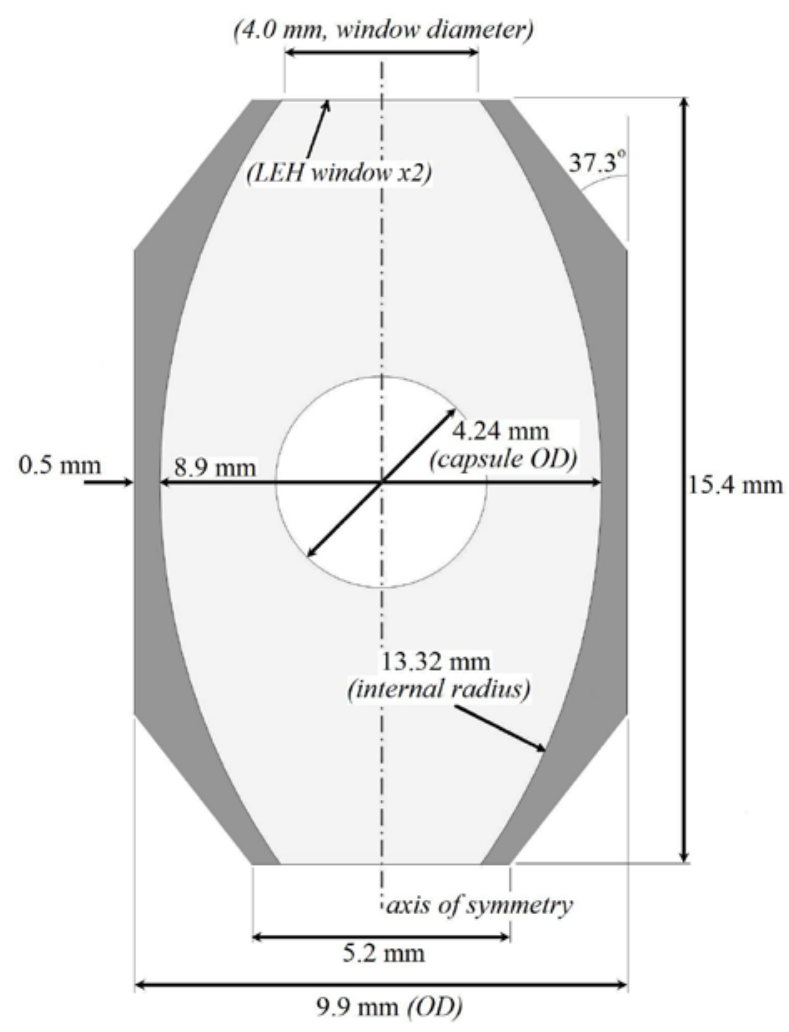

Fig. 1. LIFE.2 Hohlraum Schematic

$$
k=1.989 e^{-4} \frac{\sqrt{T / M}}{\sigma^{2} \Omega_{k}}
$$

Calculated values of viscosity and thermal conductivity have been confirmed within $1 \%$ for the temperature range of 100 to $5000 \mathrm{~K}$ when compared to the identical approach taken by Svehea (1962) (Ref. 4). These values were then extrapolated to 8200 to encompass the entire temperature range expected within the chamber, where there is not expected to be any residual xenon ionization at the time of injection.

\section{MODELING TECHNIQUES}

\section{III.A. Flow Description}

The hohlraum targets, as described in TABLE I, travel parallel to its axis of rotation through a xenon environment of sub-atmospheric pressure and elevated temperature. Using these properties, the dimensionless variables listed in TABLE II can be defined for material and transport properties of xenon.

TABLE II. Target Dimensionless Variables

\begin{tabular}{ccc}
\hline Dimensionless Variable & Formulation & TFC \\
\hline Reynolds number $(\mathrm{Re})$ & $=(\rho \mathrm{vd}) / \mu$ & 60 \\
Mach number $(\mathrm{Ma})$ & $=\mathrm{v} / \mathrm{a}=\mathrm{v} /(\gamma \mathrm{RT})^{1 / 2}$ & 0.27 \\
Knudsen number $(\mathrm{Kn})$ & $=\lambda / \mathrm{d}$ & 0.0090 \\
Prandtl number $(\mathrm{Pr})$ & $=\left(\mathrm{C}_{\mathrm{p}} \mu\right) / \mathrm{k}$ & 0.67 \\
\hline
\end{tabular}

Hohlraum flow properties can be characterized using the results from TABLE I and II. With the hohlraum's outside diameter, $d$, taken to be the system's characteristic length, $\mathrm{L}$; the Reynolds number is sub-100, necessitating laminar flow. Again taking the hohlraum's diameter as the characteristic length and mean free path calculated from DS2V to be $89.5 \mu \mathrm{m}$, a Knudsen number of 0.0090 is found. With the accepted transition from the continuum to transition regimes occurring for Knudsen number less than 0.1 , the flow can be considered well within the bounds of a viscous fluid but approaching the transition regime. Additionally, the flow is necessitated to remain subsonic with the calculated Mach number of 0.27 , however the high temperature necessitates the need to consider compressibility effects.

\section{III.B. Heat Transfer Correlations}

Heating correlations through use of the Nusselt number for cylinders traveling with its axis of symmetry parallel to the bulk flow was investigated. Unfortunately, very few studies were found to match this flow geometry, with the vast majority pertaining to cylinders in $2 \mathrm{D}$ cross flow. Results of these correlations are shown graphically in Fig. 4 of Section IV.C.

Of the correlations found, a recent study by Hadad and Jafarpur (2008) (Ref. 5) may be one of the most applicable. Focused on laminar axial flow, Hadad and Jafarpur use a semi-analytical approach to solve for the 
average heat flux over isothermal bodies with unit aspect ratio. The resulting average Nusselt number for a cylinder valid for Reynolds 1-100 is shown in Eq. (3).

$$
N u=1.5828+0.5506 \operatorname{Re}^{1 / 2} \operatorname{Pr}^{1 / 3}
$$

An older study by Belov and Terpigor'ev (1969) (Ref. 6) may be of some use for the heat transfer at the forward stagnation point. Their study was motivated by trying to account for discrepancies between experimental data and theory of heat transfer at the stagnation point of blunt bodies immersed in subsonic and supersonic jets through turbulence within the bulk flow. Within their analytical derivation lies the case of heat transfer near the stagnation point for laminar, subsonic flow, thus matching LIFE's target flight conditions, and is shown in Eq. (4).

$$
N u=0.763 \operatorname{Re}^{0.5} \operatorname{Pr}^{0.4}
$$

Kang and Sparrow (1987) (Ref. 7) conducted a series of experiments measuring the local heat transfer along the longitudinal surface of open- and closed-bore cylinders with the bulk flow parallel to its axis of rotation. The maximum heat transfer along the longitudinal surface of the closed-bore cylinder is shown in Eq. (5). This correlation is valid for the elevated Reynolds range of $7,700-47,000$ and is included to illustrate the single study found looking at heating of the hohlraum's longitudinal surface.

$$
N u=0.202 \operatorname{Re}^{2 / 3} \operatorname{Pr}^{1 / 3}
$$

An older study by Sogin (1964) (Ref. 8) measured the heat transfer at the rear of a bluff object in a lowspeed airstream. Experiments were conducted using a flat-strip spanning the length of a wind tunnel, simulating two-dimensional flow. For an angle of attack of $90^{\circ}$, heating along the back surface of the plate was found constant, represented by Eq (6). Since these experiments were conducted near atmospheric pressure, the Reynolds number range of 100,000 to 440,000 was significantly higher then for those of the LIFE heating conditions, and again was included to show the only study found looking at the heating of the rear of bluff objects.

$$
N u=0.20 \operatorname{Re}^{2 / 3}
$$

\section{III.C. ANSYS and DS2V Simulation Configurations}

The CFD code ANSYS CFX was used first in modeling the flow of xenon over the LIFE.2 target. Taking advantage of symmetry, a $45^{\circ}$ wedge $20 \mathrm{~cm}$ in length and $5 \mathrm{~cm}$ in radius was constructed with the LIFE.2 hohlraum centered along the axis of symmetry. A simplified two-dimensional representation of this domain is seen in Fig.2.

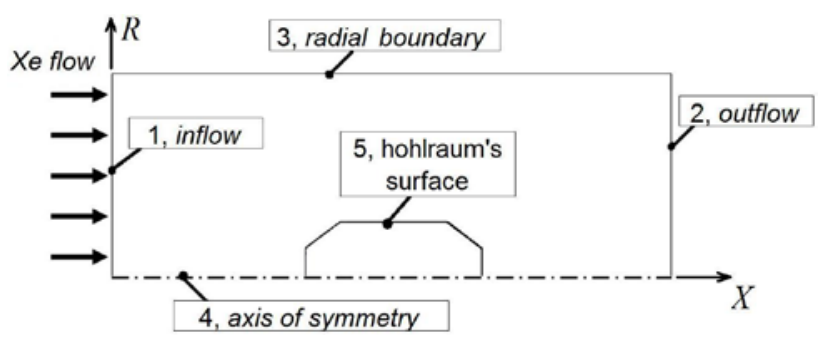

Fig. 2. Simulation Domain Schematic

Assuming the flow travels in the positive x-direction, the inflow was set to surface 1 of Fig. 2 as subsonic with normal speed and static temperature of $250 \mathrm{~m} / \mathrm{s}$ and 8000 $\mathrm{K}$. The outlet was then forced to be designated as surface 2, with an average static pressure held at $3037 \mathrm{~Pa}$, while the radial boundary of surface 3 was set as an adiabatic, free-slip wall. Surface 4 was created by designated the two lateral sides of the wedge to be symmetric, and finally the hohlraum's surface of boundary 5 was assumed to be non-slip and held at a temperature of $150 \mathrm{~K}$.

The wall was held at this elevated temperature since the xenon gas is expected to deposit onto the surface until the wall reaches its critical sublimation temperature of $127 \mathrm{~K}$. Although deposition of the xenon is not the focus of this work, holding the wall temperature at $150 \mathrm{~K}$ allows for modeling xenon properly in the gas domain. However, the nearly $8000 \mathrm{~K}$ temperature difference proved difficult for the ANSYS numerical solver to handle, and a solution was not obtained until the wall temperature was raised to $2000 \mathrm{~K}$.

In stark contrast to traditional CFD codes in which solutions are obtained that model the flow as a continuum, the direct simulation Monte Carlo (DSMC) code DS2V (Ref. 9) was used to directly simulate these physical processes through the simulation of thousands or millions of representative molecules undergoing representative collisions. The applicability of the DSMC and NavierStokes methods for the LIFE target flight conditions is coincidental, but the diversity of the two methods is beneficial in determining the accuracy of the two results.

The DS2V simulation was set up to be axial symmetric and to include radial weighting factors to help evenly distribute particles throughout the domain. Upon making this choice, boundary 4 of Fig. 4 was designated as the default axis of symmetry. The flow was then assumed to be traveling in the positive $\mathrm{x}$-direction, with boundaries 1 and 3 interfacing the reference stream where the xenon density, temperature and speed were fixed. As before with ANSYS, boundary 2 was then held at fixed pressure, and finally the hohlraum's surface of boundary 5 was fixed at a temperature of $150 \mathrm{~K}$ with full thermal accommodation. Due to computational and time 
constraints, only the first half of the LIFE.2 hohlraum was simulated.

The final solutions in heat transfer for both DS2V and ANSYS have been shown to be independent of resolution (molecular number for DS2V, mesh refinement for ANSYS) and boundary proximity.

\section{EXTERNAL CONVECTIVE HEATING OF LIFE TARGETS}

\section{IV.A. Flow Field Comparison}

Flow field contours of temperature, velocity and pressure produced by ANSYS for the elevated wall temperature of $2000 \mathrm{~K}$ are illustrated in Fig.3 (a-c). The first contour shows an increase in temperature of approximately $100 \mathrm{~K}, 3 \mathrm{~mm}$ in front of the leading edge before being quickly brought down to the $2000 \mathrm{~K}$ wall temperature. The velocity contour of Fig. 3 (b) shows a slow stagnation of the xenon gas along the axis of symmetry leading to the stagnation point, in addition to an increase of $23 \mathrm{~m} / \mathrm{s}$ when accelerated around the hohlraum's second leading edge. Consequently an increase in pressure seen near the stagnation point, as well as decrease in pressure when rounding the second leading edge, is seen in the final illustration if Fig.3 (c).

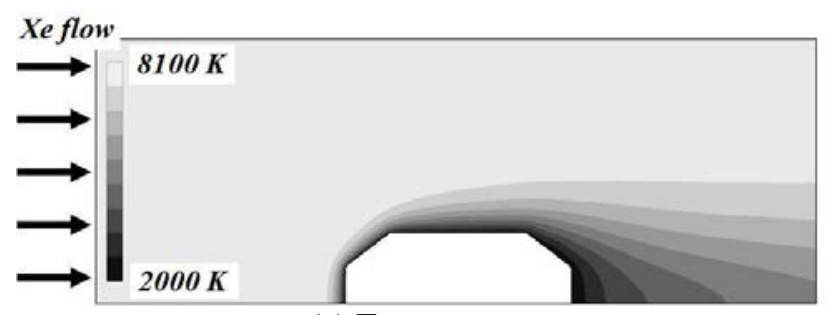

(a) Temperature

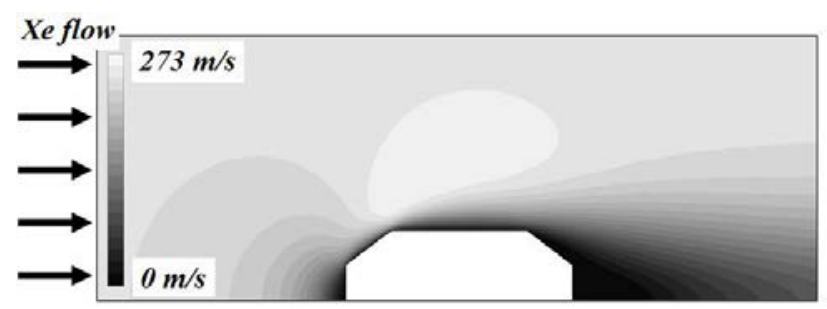

(b) Velocity

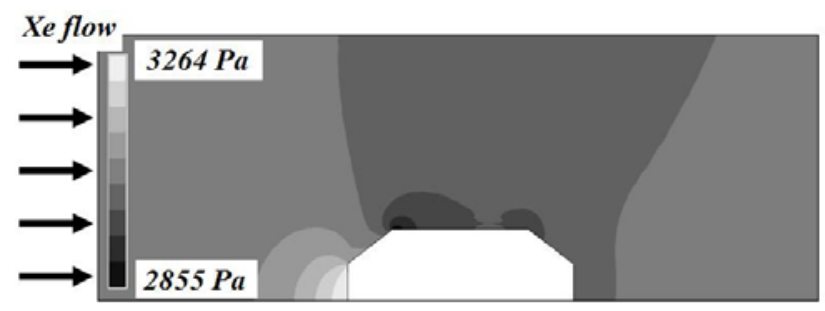

(c) Pressure

Fig. 3. ANSYS Flow Field Contours
To illustrate the differences in the two computational methods, DS2V was reran with an elevated wall temperature of $2000 \mathrm{~K}$ and compared with the temperature, velocity and pressure ANSYS profiles along the stagnation line leading up to the stagnation point in Fig. 4 (a-c).

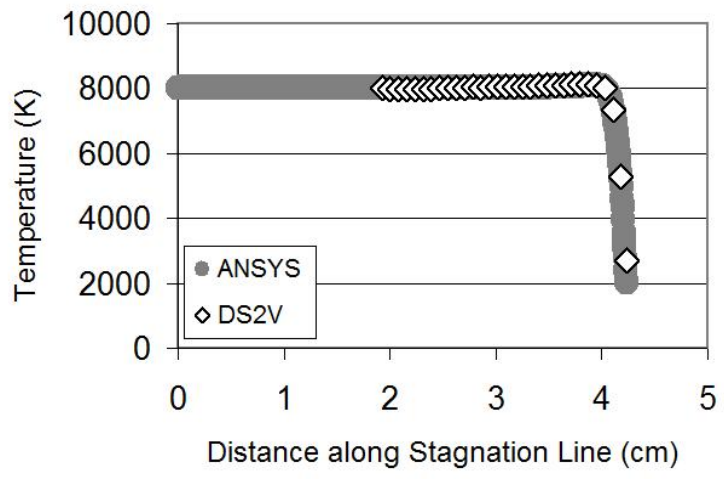

(a) Temperature

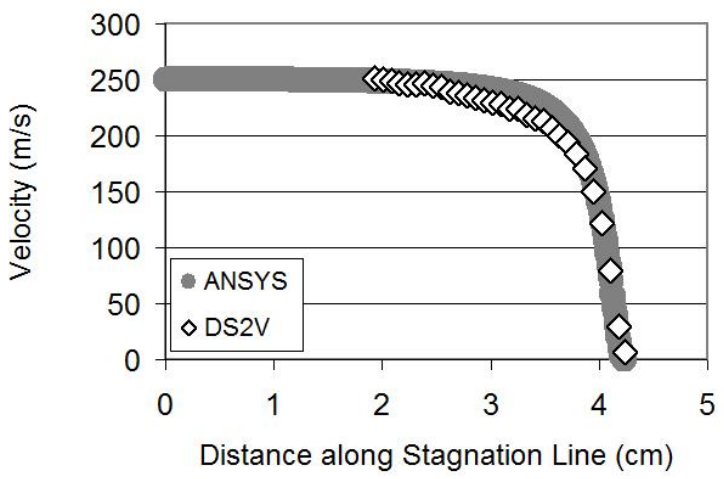

(b) Velocity

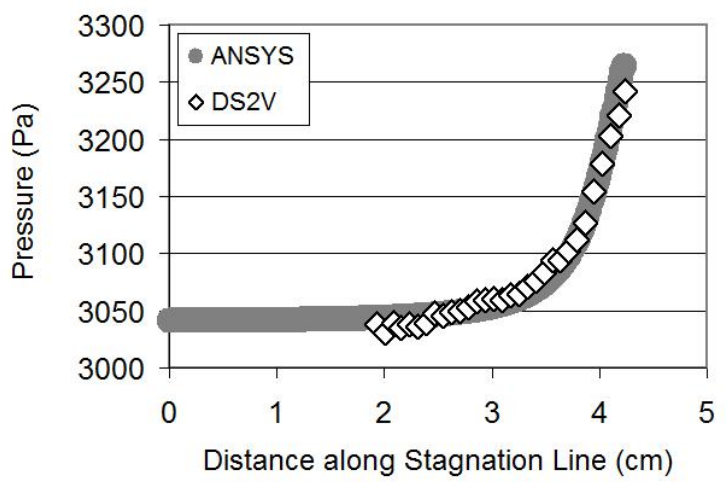

(c) Pressure

Fig. 4. Stagnation Line Property Comparison

For each of the three stagnation line property illustrations of Fig.4, excellent agreement is seen between the DS2V and ANYS solutions in both magnitude and space. There appears to be some statistical scatter in the 
DS2V solution for velocity and pressure, but this is a direct result of the lack in refinement from limited time in running DS2V at the elevated wall temperature of 2000 $\mathrm{K}$, and not of concern for the final heat transfer results of the subsequent section where excellent refinement was shown.

A useful means of comparing the increases in temperature and pressure is in calculation of the isentropic or 'total' flow properties. From Anderson (Ref. 10), the isentropic pressure, $\mathrm{p}_{t}$, and temperature, $\mathrm{T}_{\mathrm{t}}$, in terms of the overall Mach number have been calculated and are shown next to the maximum temperature and pressure simulated by ANSYS and DS2V in TABLE III.

TABLE III. Isentropic Flow Comparison $\operatorname{TFC}\left(T_{w}=2000 \mathrm{~K}\right)$

\begin{tabular}{cccc}
\hline Flowfield Property & Isentropic & DS2V & ANSYS \\
\hline Temperature $(\mathrm{K})$ & 8197 & 8121 & 8100 \\
Pressure $(\mathrm{Pa})$ & 3228 & 3242 & 3231 \\
\hline
\end{tabular}

From TABLE III, it can bee seen that both ANSYS and DS2V model a stagnation pressure very close to each other but not breaching the theoretical maximum isentropic temperature of $8197 \mathrm{~K}$. Conversely, the stagnation pressure predicted by the two codes is mildly higher than the isentropic pressure of $3228 \mathrm{~Pa}$. This clearly is not physical, and points to slight inadequacies in both ANSYS and DS2V in accurately modeling the LIFE target flight conditions.

\section{IV.B. Local Heat Flux}

The local heat flux along the surface of the hohlraum is plotted in Fig. 6 starting at the windward center ending at the leeward center. The ANSYS solution was normalized to a wall temperature of $150 \mathrm{~K}$ for a reference temperature of $8000 \mathrm{~K}$, thus matching the parallel simulation of DS2V.

Excellent agreement is seen between the simulated heat fluxes between the DS2V and ANSYS solutions, with ANSYS predicting an 8\% higher heat flux on average. Much of this difference is attributed to the larger increase in heat flux predicted by ANSYS around the edges of the hohlraum. However, this higher estimation by ANSYS may be explained in part through the presence of slip flow, measured to be approximately $7 \mathrm{~m} / \mathrm{s}$ by DS2V around the leading edge. If the hohlraum wall were exposed to velocity slip, the no-slip boundary condition of the ANSYS simulation would over constrain the solution, artificially increasing the local heat flux.

The diverse heating correlations from literature compares well with the obtained solutions of DS2V and ANSYS, giving the heat transfer to the hohlraum a sound basis. The average hohlraum heating predicted by Hadad and Jafarpur is approximately $21 \%$ lower than simulated by ANSYS. Nevertheless, this may be explained in part from their assumption of incompressibility, resulting in an overestimation of the thermal boundary layer thickness and underestimation of the heat flux to the target. The differences in shape of the LIFE.2 target to a hohlraum of unit aspect ratio will undoubtedly introduce additional uncertainty. Heating near the stagnation point predicted by Belov and Terpigor'ev proved only slightly higher than the two previous averages, but once again is based on the assumption of incompressibility. Heating along the longitudinal edge by Kang and Sparrow, in addition to the heating along the rear surface of the hohlraum have been applied well outside of their accepted bounds, and have been included for a reference to flows of higher Reynolds number.

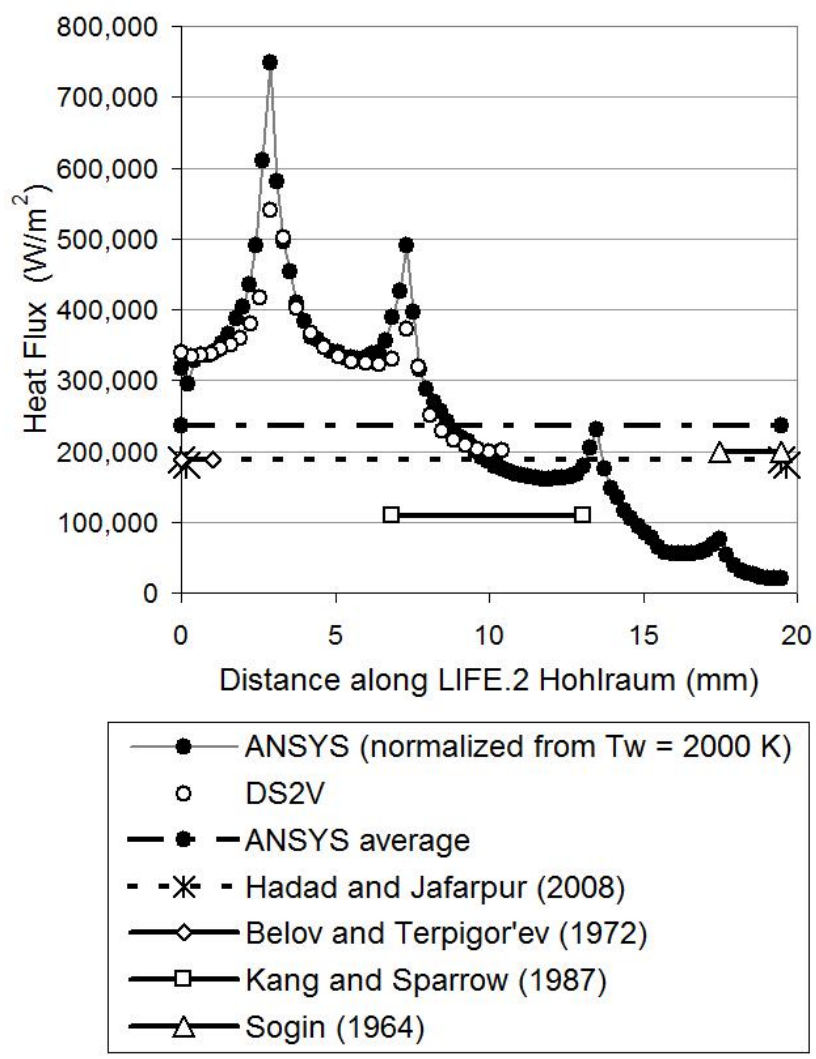

Fig. 5. Local Heat Flux along LIFE.2 Hohlraum; $\mathrm{T}_{\text {ref }}=$ $8000 \mathrm{~K}, \mathrm{~T}_{\mathrm{w}}=150 \mathrm{~K}$

\section{IV.C. Hohlraum Shaping with Baffles}

Melting of either the laser entrance hole windows or deuterium-tritium fuel layer constitutes failure of the target and no burn. To illustrate a design improvement, heating to the window was reduced, a baffle $2 \mathrm{~mm} \times 0.2$ $\mathrm{mm}$ was placed on the windward and leeward surfaces of the LIFE.2 hohlraum, and ANSYS was re-run to produce the velocity flow field shown in Fig. 6. 


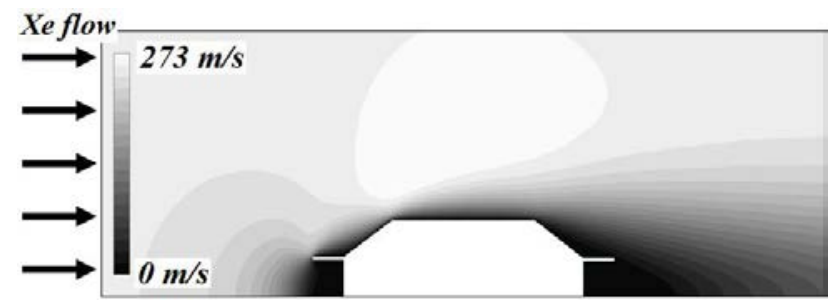

Fig. 6. Velocity Profile with 2 mm Baffles

While the size of the baffle may be excessive in length due to the blocking of incident laser beams, the heat flux to the windward LEH window was reduced by a factor of 4.3! This pitot-static tube inspired design effectively traps xenon gas near the LEH window, reducing the convective heat transfer mechanism. In addition, since the target is expected to be in free-flight in an ambient xenon environment near room temperature prior to reaching the target chamber, the xenon gas trapped near the window would have to come up to temperature before being able to heat the window, further delaying its heating when compared to the steady state solution depicted in Fig. 6. Finally, the presence of this baffle would effectively shade the LEH window from additional radiative heating from the LIFE chamber walls, a heat transfer mechanism not studied within this work.

\section{CONCULSIONS AND RECOMMENDATIONS}

Making use of direct simulation Monte Carlo and Navier-Stokes CFD methods, the heating of LIFE.2 targets traversing the target chamber has been analyzed. The simulation codes DS2V and ANSYS provided the ability to study the convective heat transfer from the hightemperature xenon gas of the chamber to the LIFE hohlraums and provide consistent flow field solutions and local heat flux to the target's surface. Empirical and analytical heating results produced consistent results with the code solutions, while the codes themselves closely matched each other.

Based on the results of the work herein, it is recommended the LIFE hohlraum include baffles on the forward and leeward surfaces. These baffles have been shown to trap xenon near the surface of the sensitive LEH windows, reducing the effects of convective heat transfer. In addition, the presence of the baffles will act to shade the interior of the hohlraum from radiation coming from the chamber walls, further improving target survival independent of all other reactor parameters.

\section{REFERENCES}

1. United States, Department of Energy, "OSIRIS and SOMBRERO Inertial Fusion Power Plant Designs,” Vol. 2, WJSA-92-01, DOE/ER/54100-1 (1992).

2. B. CHRISTENSEN, A. R. RAFFRAY, M. S. TILLACK, Fusion Science \& Technology, 47.4, 1175-9 (2005).

3. R. B. BIRD, W. E. STEWART and E. N. LIGHTFOOT, Transport Phenomena, $2^{\text {nd }}$ ed., Wiley, New York, NY (2007).

4. R.A. SVEHLA, "Estimated Viscosities and Thermal Conductivities of Gases at High Temperatures,” NASA TR R-132, Lewis Research Center, Cleveland, $\mathrm{OH}$ (1962).

5. Y. HADAD and K. JAFARPUR, Proc. of $6^{\text {th }}$ IASME/WSEAS International Conference on Heat Transfer, Thermal Engineering and Environment (THE’08), Greece, Rhodes, August 20-22 (2008).

6. I. A. BELOV and V. S. TERPIGOR'EV, InzhenernoFizicheskii Zhurnal, 17.6, 1106-109 (1969).

7. S. S. KANG and E. M. SPARROW, Transaction of the ASME. Journal of Heat Transfer, 109.2, 314-20 (1987).

8. H. H. SOGIN, Transactions of the ASME. Journal of Heat Transfer, 86, 200-2 (1964).

9. DS2V Version 4.5.06, GAB Consulting.

10. J. D. ANDERSON, Fundamentals of Aerodynamics, pp. 449, $3^{\text {rd }}$ ed., McGraw-Hill, Boston, MA (2001).

This work performed under the auspices of the U.S. Department of Energy by Lawrence Livermore National Laboratory under Contract DE-AC52-07NA27344. 